\title{
Optical properties of coupled metallic nanorods for field-enhanced spectroscopy
}

\author{
J. Aizpurua, ${ }^{1,2, *}$ Garnett W. Bryant, ${ }^{1, \dagger}$ Lee J. Richter, ${ }^{1}$ and F. J. García de Abajo ${ }^{2,3}$ \\ ${ }^{1}$ National Institute of Standards and Technology, 100 Bureau Drive, Gaithersburg, Maryland 20899, USA \\ ${ }^{2}$ Donostia International Physics Center, Paseo Manuel de Lardizabal 4, 20018 Donostia, Spain \\ ${ }^{3}$ Centro Mixto CSIC-UPV/EHU, Apartado Postal 1072, 20080 San Sebastian, Spain \\ Brian K. Kelley and T. Mallouk \\ Department of Chemistry, 152 Davey Laboratory, Pennsylvania State University, University Park, Pennsylvania 16802, USA
}

(Received 30 December 2004; published 28 June 2005)

\begin{abstract}
The optical properties of coupled metallic nanorods are studied to investigate the use of coupled plasmonic structures in field-enhanced spectroscopies. Light scattering by coupled nanorods is calculated with the boundary element method, including retardation. The modes of coupled nanorod systems are calculated by the boundary charge method and discussed in terms of their symmetry. Similar scattering behavior for isolated nanorods and pairs of nanorods can mask the very different local responses that produce near-field enhancement. The response of isolated rods redshifts with increasing rod length because intrarod restoring forces are reduced. The near- and far-field responses increase monotonically with increasing rod length (increasing polarization along the rod). For coupled nanorods, coupling localizes charge at the gap between the rod ends and splits degenerate modes. The localized charge depolarizes the intrarod response and provides an additional redshift. Moreover, the near-field enhancement in the gap between the nanorods is dramatically increased by coupling-induced charge localization at the gap. For short nanorods, the near-field response in coupled systems is determined by the geometry of the rod ends that define the gap. For longer nanorods, the response in coupled systems is determined by the rod length. Changing the dimensions and geometry of the nanorods to modify the interrod coupling has a major effect on the local-field enhancement. The effects of the environment and the actual metallic material do not have as big an influence on the field enhancement.
\end{abstract}

DOI: 10.1103/PhysRevB.71.235420

PACS number(s): 78.67. $-\mathrm{n}, 61.46 .+\mathrm{w}, 73.20 . \mathrm{Mf}$

\section{INTRODUCTION}

The optical properties of molecules can be influenced dramatically by coupling to the plasmon resonances of nearby metallic structures. This coupling can lead to harmful effects, such as the quenching of fluorescent molecules by metallic films, ${ }^{1}$ or to useful effects, such as surface-enhanced Raman scattering ${ }^{2,3}$ (SERS) and tip-enhanced scattering. ${ }^{4}$ The demonstration of single-molecule sensitivity ${ }^{5-7}$ via SERS has prompted a renewed interest in the electromagnetic response of nanostructured metallic systems. A consensus is emerging that the extreme enhancement of the optical field required to observe single-molecule Raman scattering occurs in the gaps between metal nanoparticles. ${ }^{5,8-10}$ This revived, strong interest in metallic nanostructures has stimulated the synthesis of more complex structures ${ }^{11-18}$ to enhance local fields on the nanoscale. ${ }^{19}$ A number of experimental efforts have recently characterized the far-field scattering of coupled metal nanoparticles, either pairs or two-dimensional (2D) arrays. ${ }^{20,21}$ However, in virtually all of these studies, the gap between the particles was limited to dimensions $\gtrsim 20 \mathrm{~nm}$ because of constraints in the lithographic fabrication.

In order to achieve the nanometer gaps needed for singlemolecule SERS, chemical-fabrication techniques must be applied to fabricate the metallic nanostructures. For example, template synthesis of metal nanorod junctions, with the junction between rods defined by single monolayers of spacer molecules, has been developed for the electrical characterization of monolayers. ${ }^{22,23} \mathrm{~A}$ template-grown nanorod twin structure, consisting of two $80 \mathrm{~nm}$ diameter Au rods sepa- rated by $\approx 2 \mathrm{~nm}$ by the presence of an oligophenyleneethynylene monolayer, is shown in Fig. 1. The rod lengths are $\approx 500$ and $\approx 200 \mathrm{~nm}$. Convex ends were created by using a glass-lined template. Template synthesis techniques provide one example where precise control over the nanorod diameter (template diameter), nanowire length (growth time), end curvature (template chemistry), and junction width (monolayer composition) should be possible.

The physics underlying the electromagnetic response of such systems is understood, ${ }^{24}$ but there are still many aspects of the enhancement that must be elucidated to develop opti-

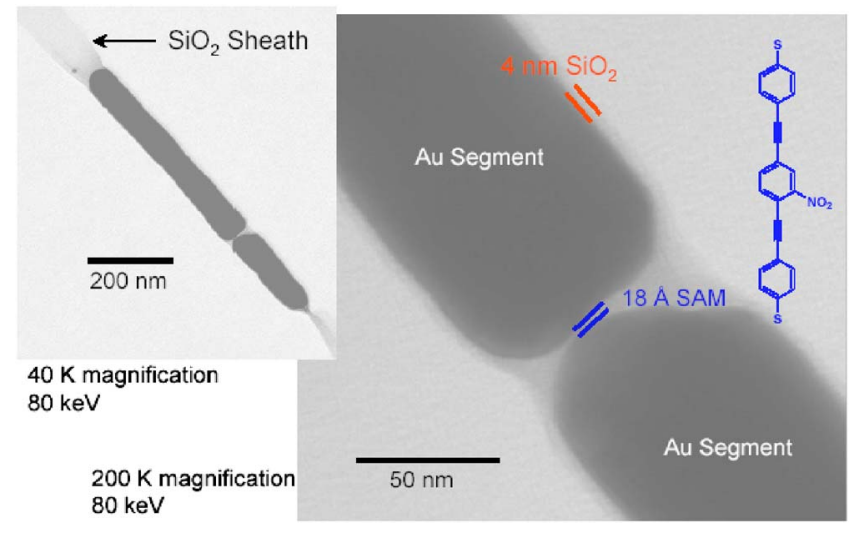

FIG. 1. SEM images of a template-grown Au nanorod pair. The higher-magnification image shows the $2 \mathrm{~nm}$ gap between the nanorods. 
mal nanostructures for field-enhanced spectroscopy. To understand the optical response of such strongly coupled metallic nanorods, here we present calculations of the optical response of isolated and coupled nanorod structures. The pioneering studies of the optical response of a sphere by $\mathrm{Mie}^{25}$ and more recent studies dealing with various nanoparticles, ${ }^{26-29}$ complex nanoshells, ${ }^{30}$ nanorings, ${ }^{31}$ and aggregates of particles, ${ }^{20,21,32-35}$ even including the use of density-functional techniques to obtain the response of the system, ${ }^{36}$ show that classical electromagnetic theory gives a reasonable description of the optical response of metallic nanostructures.

We have performed detailed calculations of the classical electromagnetic response of coupled Au nanorods to better define the rod diameter, rod length, and gap distance needed for optimal coupled structures. Both the far- and near-field responses are determined. To date, other authors have reported the optical response, ${ }^{37}$ plasmon relaxation dynamics, ${ }^{38}$ and coupling with metallic surfaces ${ }^{39}$ for isolated nanorods. Here we present results for isolated nanorods to better understand the response of coupled nanorods. Such a comparison is important. Although isolated and coupled nanorods have similar optical response, there are important differences in their local response and polarization that govern their optical properties. In addition, we determine the modes underlying the optical response of isolated and coupled nanorods and use these modes to characterize the optical response at different wavelengths. ${ }^{40,41}$ Other materials, such as silver and aluminum, are also considered to determine the effect of material response.

\section{CALCULATION METHOD}

The optical response of nanorods is calculated by means of the boundary element method in a full electromagnetic calculation, ${ }^{42}$ including retardation. Retardation must be included because we consider structures with lengths that can be larger than a wavelength. Maxwell's equations for inhomogeneous media with sharp boundaries are solved in terms of charges and currents distributed on the surfaces and interfaces. Boundary conditions are imposed via surface integrals along the boundaries between different media. Each region is characterized by a local dielectric function. The external fields interact self-consistently with the induced boundary charges and currents, which are determined by discretizing the surface integrals and solving the appropriate matrix equations. In this approach, the scattered field due to an incident external field is calculated directly. We calculate, in this way, both the near and far fields for a given structure.

To illustrate the optical response of coupled nanorods, we also calculate the modes of isolated and coupled metallic nanorods. We determine the modes in the electrostatic limit by solving the Laplace equation with the use of the boundary charge method. ${ }^{43}$ Full calculations (done with retardation) and electrostatic calculations (done without retardation) predict the same general trends and dependences on geometry, although a direct quantitative comparison between the two approaches can be complicated by the large redshifts because of retardation (see, for example, the comparison in Ref. 42).
In the full calculation, both surface-charge and surfacecurrent densities must be determined to characterize a mode. In the nonretarded limit, only a surface-charge density is needed to characterize a mode. Here, we do the mode analysis in the simpler electrostatic limit because this limit gives a reasonable, qualitative description that provides a good, more intuitive understanding of the full optical response.

In the electrostatic limit, when the materials inside and outside the nanosystem are homogeneous, the eigenvalues $\Lambda_{i}$ are calculated from the eigenequation obtained by imposing the boundary conditions ${ }^{43}$

$$
\Lambda_{i} \sigma_{i}(\mathbf{s})=-\int_{S} d s^{\prime} \frac{\mathbf{n}_{\mathbf{s}} \cdot\left(\mathbf{s}-\mathbf{s}^{\prime}\right)}{\left|\mathbf{s}-\mathbf{s}^{\prime}\right|^{3}} \sigma_{i}\left(\mathbf{s}^{\prime}\right),
$$

where $\sigma_{i}\left(\mathbf{s}^{\prime}\right)$ is the surface-charge density, $\mathbf{s}$ and $\mathbf{s}^{\prime}$ are space vectors for points on the surfaces $S$ of the rods, the integral is a surface integral over points $\mathbf{s}^{\prime}$ on $S$, and $\mathbf{n}_{\mathbf{s}}$ is the normal vector to the surface at point $\mathbf{s}$. We obtain a series of eigenvalues that depend on the geometry of the nanostructure. The particular energy $\hbar \omega$ or wavelength $\lambda$ of the mode is obtained by means of the functional relationship that the eigenvalue $\Lambda_{i}$ and the dielectric functions fulfill

$$
\Lambda_{i}=2 \pi \frac{\epsilon_{2}(\omega)+\epsilon_{1}(\omega)}{\epsilon_{2}(\omega)-\epsilon_{1}(\omega)} .
$$

The dielectric functions $\epsilon_{1}$ and $\epsilon_{2}$ are the $\omega$-dependent dielectric functions inside and outside the structure (as defined by $\mathbf{n}_{\mathbf{s}}$ ). For dielectric functions, we use tabulated data in the literature (see Ref. 44).

\section{LIGHT SCATTERING BY ISOLATED NANORODS}

First, we discuss the response of isolated nanorods so that we can better understand the response of coupled systems. We consider single cylindrical gold nanorods with a radius $R=40 \mathrm{~nm}$ and hemispherical, rounded ends, similar to the rods shown in Fig. 1. The rods are surrounded by vacuum $\left(\epsilon_{2}=1\right)$. We determine both the far- and near-field responses for a plane-wave incident perpendicular to the nanorod with the light polarized parallel to the rod axis. The far-field observation point is in the plane defined by the rod axis and propagation direction [see the schematic in Fig. 2(a)]. The dependence of the far-field intensity on the incident-field wavelength $\lambda$ for different lengths $L$ of the nanorods is shown in Fig. 2. Here, $L$ is defined as the length of the cylindrical section of the nanorod. For a sphere (nanorod with $L=0$ ), a peak in the far-field response is seen at the wavelength $\Gamma_{\text {sphere }} \approx 510 \mathrm{~nm}$. $\Gamma_{\text {sphere }}$ is independent of sphere radius for small spheres and redshifts slightly, because of retardation, for large spheres. ${ }^{45}$ For small $L$, a single peak is seen at the wavelength $\Gamma_{1}$. As $L$ increases, this peak redshifts to the near infrared. The $L$ dependence of $\Gamma_{1}$ is shown in Fig. 3. A similar linear $L$-dependence is obtained in nonretarded calculations for nanorods. ${ }^{37}$ Our results for nanorods, which include the effects of retardation, are redshifted from the nonretarded limit. This long-wavelength peak results from dipolar longitudinal charge oscillation ${ }^{37}$ along the rod axis, which is consistent with the mode charge distribution shown 

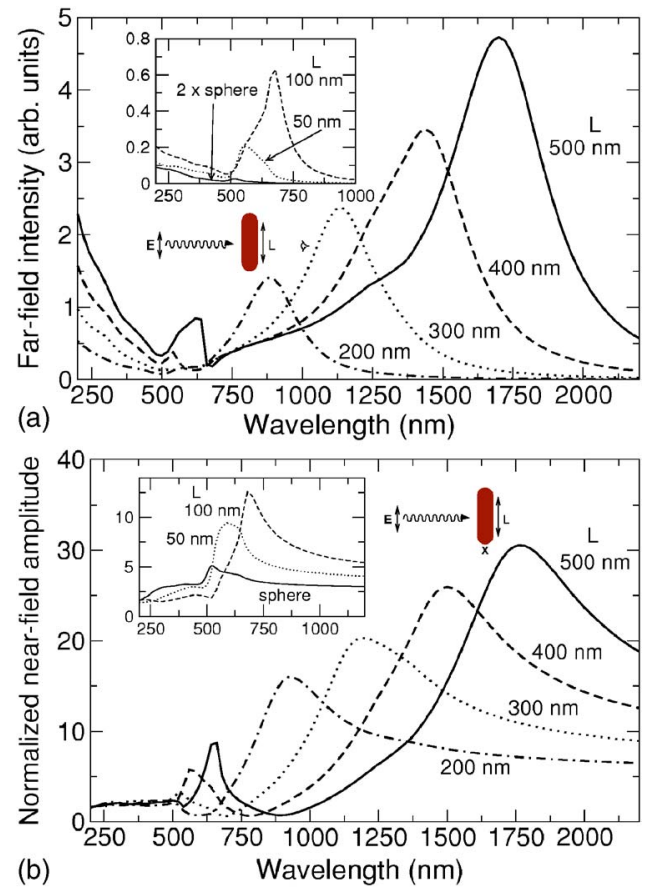

FIG. 2. (a) Far-field intensity as a function of wavelength for an electromagnetic plane wave incident on a cylindrical Au nanorod as depicted in the schematic. The polarization is parallel to the rod. $L$ is the length of the nanorod. The rod radius $R$ is $40 \mathrm{~nm}$. All figures of far-field intensity use a common (but arbitrary) scale. (b) Normalized near-field amplitude for the system as in (a). The near field is evaluated $1 \mathrm{~nm}$ from the nanorod. Results for small $L$ are shown in the insets.

in Fig. 4 and discussed below. For this mode, the half wavelength of the dipolar charge oscillation is slightly longer than $L$ because of end effects; that is, $L \approx \Gamma_{1} / 3$. This peak redshifts with increasing rod length because the separation between the dipole charges increases, thereby reducing the restoring force that determines the oscillation frequency.

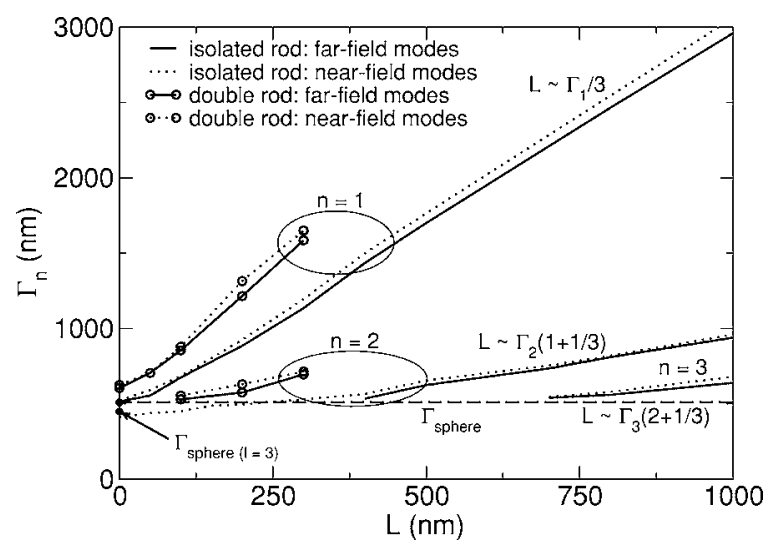

FIG. 3. Dependence of feature wavelength $\Gamma_{n}$ on nanorod length $L$ both for isolated $\mathrm{Au}$ rods and for two coupled identical Au rods that are separated by $2 \mathrm{~nm}$. $R=40 \mathrm{~mm}$. Feature wavelengths for both far- and near-field response are shown. The incident field is polarized parallel to the nanorod axis. The limit $\Gamma_{\text {sphere }}$ is indicated. The limit for an $l=3$ spherical plasmon mode, expected if the sphere dipole mode has wavelength $\Gamma_{\text {sphere }}$, is also indicated.
Because the effective dipole moment increases with increasing $L$, the strength of this peak increases monotonically with increasing $L$. Up to the largest $L$ that we have considered $(1500 \mathrm{~nm})$, this peak wavelength and peak far-field intensity both increase monotonically with $L$. Retardation does not suppress the response for long rods because the peak wavelength $\Gamma_{1}$ is a factor of $\sim 3$ greater than $L$.

For longer nanorods, additional features in the far-field response at wavelengths $\Gamma_{n}$ above $\Gamma_{\text {sphere appear first as }}$ shoulders and then become peaks with increasing $L$. These features also redshift with increasing $L$. (A feature seen near $650 \mathrm{~nm}$ is due to a kink in the empirical dielectric constant used for Au. ${ }^{44}$ This feature is independent of nanorod size and interferes with the plasmonic features that redshift through this wavelength. A similar feature near $1350 \mathrm{~nm}$ arises for the same reason.) The additional plasmonic features are higher-order dipolar oscillations along the rod. For example, the second feature, with $L \approx 4 \Gamma_{2} / 3$, corresponds to the next dipole-active (antisymmetric) mode that has approximately an odd number of half wavelengths in the charge oscillation. For the nanorods shown in Fig. 2, this higher mode produces the second, higher-energy, shorterwavelength peak between 525 and $650 \mathrm{~nm}$. This peak is less intense than the peak for the lowest mode for incoming light polarized along the rod axis, indicating that this higher mode has a smaller dipole moment and less net charge buildup at the ends of the rod than the lowest dipole mode, consistent with the modes shown in Fig. 4.

For incident light polarized perpendicular to the rod axis, there is little far-field response near $\Gamma_{1}$ because the longitudinal charge oscillation cannot be driven by this polarization. There is significant far-field response from the higher modes, indicating that they provide both longitudinal and transverse oscillations. However, the response of higher modes to perpendicular polarization is still weaker than the response to parallel polarization because of the smaller transverse dipole moments that can be excited. Just above $\Gamma_{\text {sphere }}$, the far-field response to perpendicular polarization is slightly blueshifted from the corresponding far-field response to parallel polarization because the perpendicular polarization excites more efficiently, higher-energy, more transverselike modes.

In the very short-wavelength region (i.e., the region below $\Gamma_{\text {sphere }}$ ), we observe a rising background for the far-field response connected with bulk plasmons, radiating surface plasmons, which should occur at energies above the bulk plasmon of the material, ${ }^{46}$ higher-order rod modes at wavelengths below $\Gamma_{\text {sphere, }}$, and interband transitions in $\mathrm{Au}$ that occur below $500 \mathrm{~nm} .{ }^{47}$ As a result of the damping from interband transitions, the plasmonic features in the far-field response below $500 \mathrm{~nm}$ are significantly broadened and not identifiable. These contributions overlap at these shorter wavelengths, making it difficult to identify specific contributions. This background scales approximately with the size of the rod without significant shifts of the far-field response, further indicating the importance of the bulklike response.

To better identify the contribution of the interband transitions, we have also calculated the nanorod response by setting the imaginary part of the Au bulk dielectric function to be small for wavelengths below $500 \mathrm{~nm}$. When the interband effects are reduced in this manner, the far-field response just 


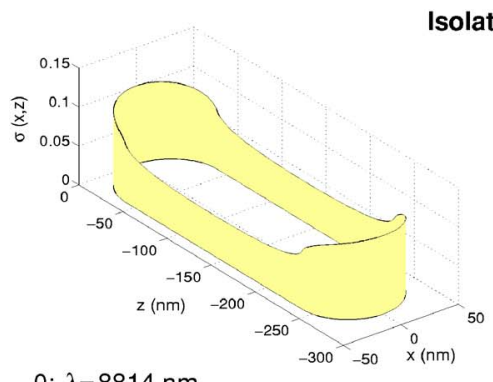

$0: \lambda=8814 \mathrm{~nm}$
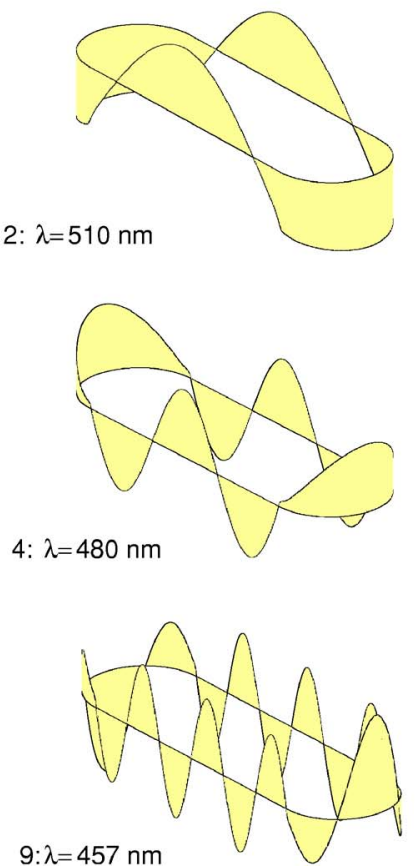

Isolated rod
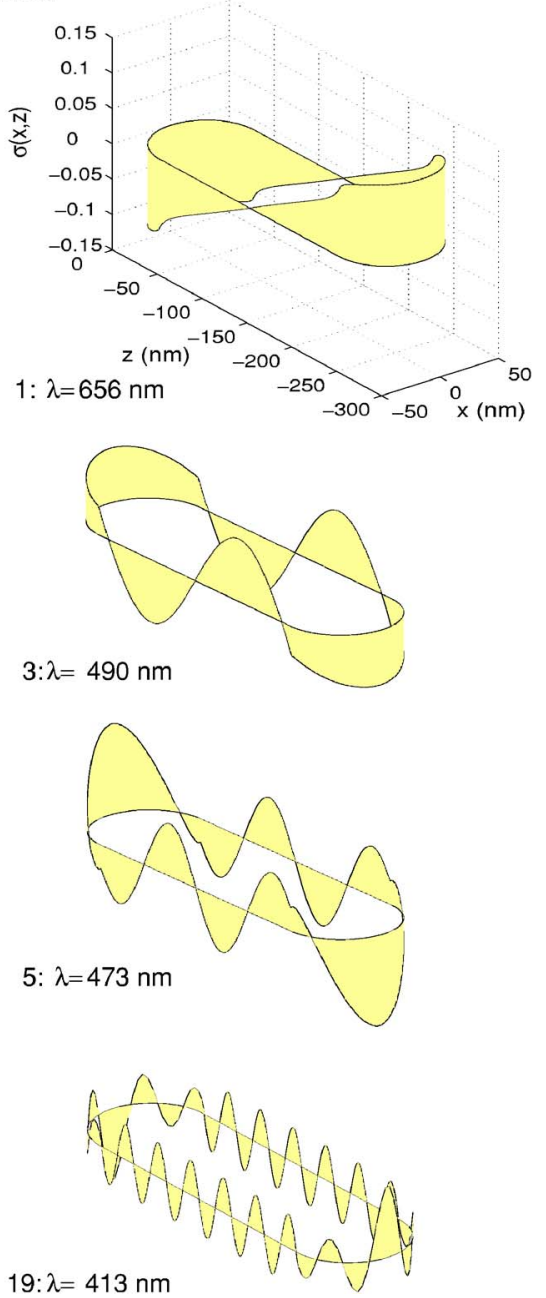

FIG. 4. Cross sections of the surface-modes surface-charge density in an isolated gold nanorod. $L=200 \mathrm{~nm}, R=40 \mathrm{~nm}$. These modes have $m=0$ azimuthal number, so the surface-charge density is cylindrically symmetric around the rod axis. The bulk and surface plasmon wavelengths in gold are 226 and $392 \mathrm{~nm}$, respectively. below $500 \mathrm{~nm}$ is significantly weaker, while the response near $200 \mathrm{~nm}$ from radiating plasmons remains sizable. Features due to rod modes below $500 \mathrm{~nm}$ become more apparent when the interband damping is reduced.

The near-field amplitude on the rod axis, $1 \mathrm{~nm}$ outside the end of the nanorod and normalized to the incident field at this point, is shown in Fig. 2(b). The normalized near field shows features similar to those in the far field, with $\Gamma_{n}$ and the field enhancement increasing monotonically with increasing $L$. Near-field enhancements of 10-70 (for $L$ $=1500 \mathrm{~nm}$ ) are seen for isolated rods. However, the nearfield features are slightly redshifted from the corresponding far-field features and have different relative weights. The farfield response also has sharper long wavelength peaks.

The near-field response below $\Gamma_{\text {sphere }}$ is weak, indicating that the bulklike response below $\Gamma_{\text {sphere }}$ contributes weakly to the near field. Because the interband damping has less effect on the near-field response, broad plasmonic (lengthdependent) features are discernible in the weak near-field response below $\Gamma_{\text {sphere. }}$ As shown in Fig. 3, the feature for $\Gamma_{2}$ can be followed back to the sphere limit $(L=0) . \Gamma_{1}$ clearly evolves from the lowest dipole-active (angular momentum $l=1)$ spherical plasmon mode. $\Gamma_{2}$ evolves from a higherorder spherical plasmon, presumably the next dipole-active $(l=3)$ spherical plasmon. (Far-field features cannot be clearly identified below $\Gamma_{\text {sphere }}$, therefore they are not shown in this region. Similarly, $\Gamma_{3}$ cannot be followed clearly down to the $L=0$ limit.)

To further understand the excitations produced in the nanorods, we calculate the surface plasmon modes in these structures by means of the boundary charge method in the electrostatic limit. The features $\Gamma_{n}$ in the far- and near-field responses should correlate with the dipole-active modes. The surface charge distributions $\sigma(\mathbf{s})$ for the lowest modes of an isolated gold nanorod are shown in Fig. 4 for azimuthal number $m=0$. In the electrostatic limit, the wavelengths $\lambda$ of the modes are obtained from the $\omega$ that solve Eq. (2) with use of the eigenvalues of Eq. (1) and the tabulated data for gold. ${ }^{44}$ The lowest mode is a zero-order mode, which does not have any oscillation along the rod axis. This mode has finite charge, is connected with infinitely long wavelength oscillations, and cannot be excited by an external electromagnetic wave. The first mode with physical meaning is a dipolar mode, with a nodal ring around the rod axis at the center of the rod, which piles up positive and negative charge at opposite ends of the nanorod. This mode responds to a field polarized along the axis and drives the response at long wavelength (the $n=1$ peak). The low-order $m=0$ modes can be identified by the number of nodal rings around the rod axis. Even-order modes have an even number of nodal rings 
and no net dipole in their charge distribution. Odd-order modes, with an odd number of nodal rings and an odd number of half wavelengths, always have charges of opposite sign at the nanorod ends, giving a net dipole. For both types of modes, the wavelengths decrease as additional longitudinal nodes are added. For higher-order modes, the surfacecharge oscillation includes nodes on the rounded ends of the rod. These modes have partial transverse character in the oscillation pattern and can be excited by transverse fields. These modes are connected with the features observed just above $\Gamma_{\text {sphere }}$ and with the weak structure observable below $\Gamma_{\text {sphere }}$ in the near-field response. They are also present in the response to an incident wave with longitudinal polarization, both because these modes have mixed longitudinal and transverse character and because end effects can provide a coupling of transverselike modes to a longitudinal polarization.

As seen in Fig. 4, the surface charge of each mode has a kink where the hemispherical cap begins, such that the local effective wavelength of the charge oscillation is longer on the cap than on the cylindrical shaft of the rod. This suggests a local slowing down of the charge oscillation due to the charge buildup at the ends. ${ }^{48}$

\section{LIGHT SCATTERING BY COUPLED NANORODS}

To investigate the effects of coupling between nanorods, we consider pairs of nanorods aligned along a common axis as in the experimental setup described in the introduction. We first present the far- and near-field responses for a plane wave incident on a pair of gold cylindrical nanorods, each $200 \mathrm{~nm}$ long and $40 \mathrm{~nm}$ in radius. Each end of the nanorods is rounded with a hemispherical cap. The light is incident normal to the axis of the rods with polarization along the rod axis. The far field is calculated as for the isolated rod, whereas the normalized near field is calculated along the rod axis between the rods.

Figure 5 shows the change in the far- and near-field responses at the midpoint between the rods when the separation $S$ between rods is varied to modify the coupling. In general, the same features are seen for isolated and coupled rods. There is a long-wavelength peak because of the lowest dipole-active mode. The position $\Gamma_{1}$ of this peak redshifts by $300 \mathrm{~nm}$ when the gap $S$ is reduced to $2 \mathrm{~nm}$ because of the depolarization of the intrarod charge oscillations induced by the coupling. As discussed later (see Figs. 6 and 10), opposite charges are strongly localized to the ends of the two rods at the gap when the rods are coupled. Strong attraction across the gap competes against the intrarod, restoring forces that drive the intrarod charge oscillations and provides this additional redshift. ${ }^{20,21,33}$ As for the isolated rod, the near-field response is redshifted slightly from the far-field response for coupled rods.

Figure 7 summarizes the gap dependence of $\Gamma_{1}$ for the far-field response, and for the near-field response both in the gap $1 \mathrm{~nm}$ from the end of a rod and at the midpoint of the gap. The near-field response is redshifted from the far-field response at all $S$. If the gap is small, the near-field response at any point in the gap along the rod axis is nearly the same, with the near-field response at midgap slightly redshifted
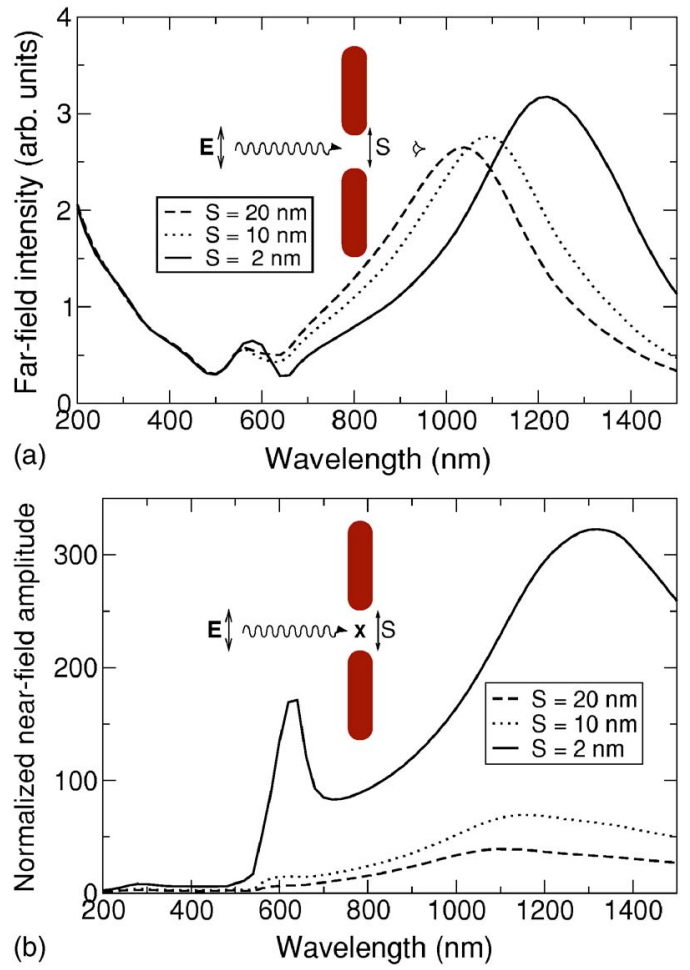

FIG. 5. (a) Far-field intensity as a function of wavelength for a plane wave incident on a pair of identical cylindrical Au nanorods, as shown in the schematic. $S$ is the separation distance between the nanorods. $L=200 \mathrm{~nm}, R=40 \mathrm{~nm}$. The polarization is parallel to the rod. (b) Normalized near-field amplitude at the midpoint between two nanorods for the same geometry as in (a).

from the response $1 \mathrm{~nm}$ from a rod end. For large gaps, the near-field response at different points along the axis in the gap can be significantly different, with $\Gamma_{1}$ at the midgap substantially redshifted from the $\Gamma_{1}$ for the response $1 \mathrm{~nm}$ from an end. For large gaps, $\Gamma_{1}$ for the far-field response and for the near-field response $1 \mathrm{~nm}$ from the rod end both oscillate about the appropriate limits for an isolated rod, with the oscillation slowly damping as $S$ increases. This oscillation reflects an oscillation in the sign of the coupling between rods because of retardation when the gap is greater than a

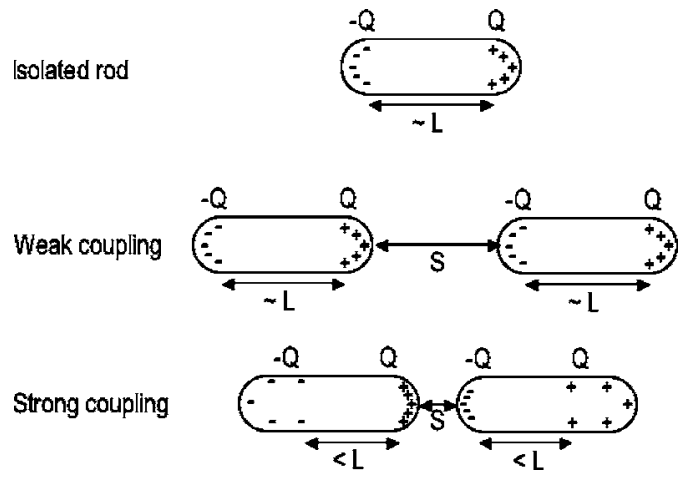

FIG. 6. Model of the expected surface-charge distribution that drives the long-wavelength dipolar response of an isolated rod, of two widely spaced, weakly coupled rods, and of two closely spaced, strongly coupled rods. 

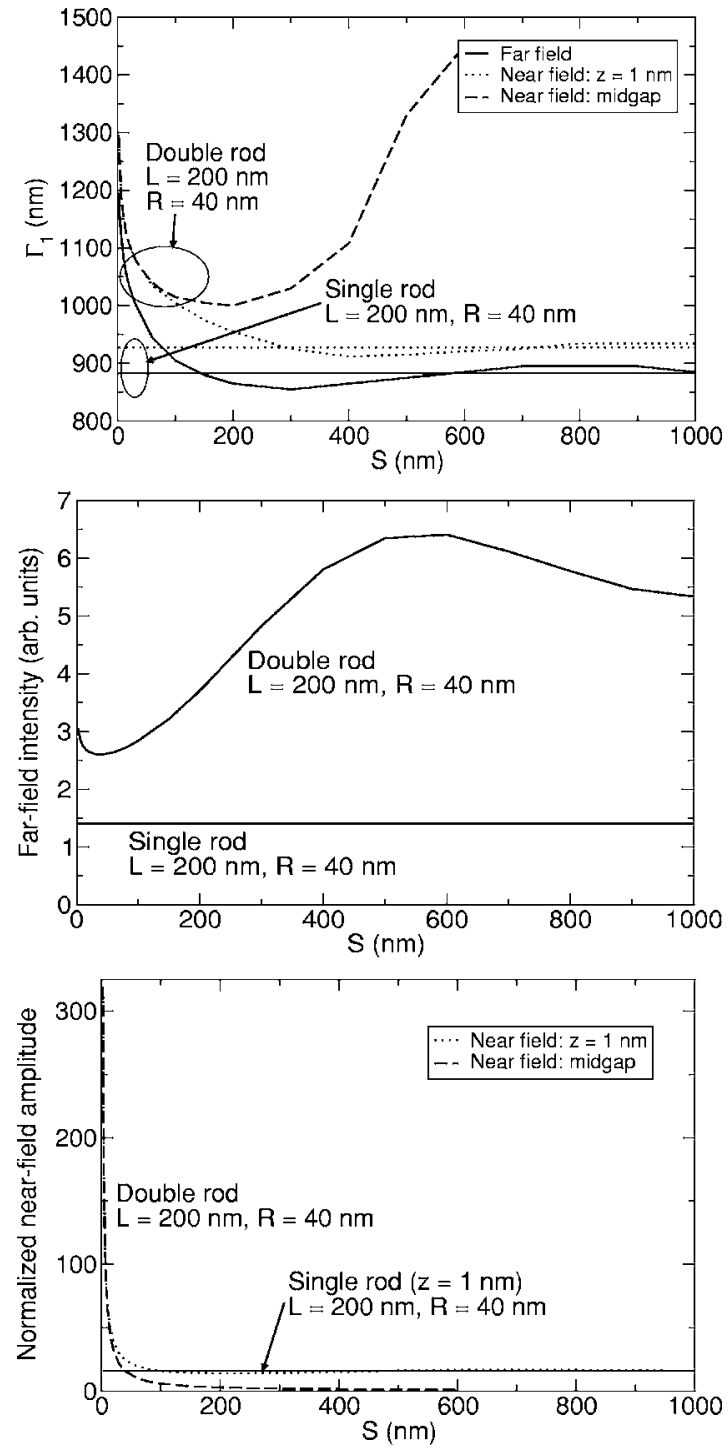

FIG. 7. Dependence of the far-field and near-field response of a pair of coupled identical Au nanorods on the interrod gap $S$ for a plane wave incident with polarization parallel to the rods. $L$ $=200 \mathrm{~nm}, R=40 \mathrm{~nm}$. The peak wavelength $\Gamma_{1}$ for the dipolar response, the far-field intensity at $\Gamma_{1}$, and the normalized near-field amplitude at $\Gamma_{1}$, both at midgap and in the gap $1 \mathrm{~nm}$ from the end of a rod, are shown. The corresponding results for an isolated rod are shown.

quarter wavelength. ${ }^{21}$ For large $S$, the field at midgap is small. We have not followed the $S$ dependence of $\Gamma_{1}$ at midgap beyond this point.

The $S$ dependence of the intensity of the long-wavelength, dipolar far-field response (see Fig. 7) can be understood with the simple coupled dipole picture illustrated in Fig. 6. If an incident field induces a charge $\pm Q$ at the ends of an isolated rod, then almost the same charges $\pm Q$ should be induced at the ends of two widely spaced, weakly coupled rods. The dipole moment due to the charge at the two outside ends of the coupled rod structure is roughly $Q(2 L+S)$ [here the length of the rod caps is ignored for simplicity]. The dipole moment due to charge at the gap is $-Q S$. The net dipole moment is $\sim 2 Q L$, twice the dipole moment of an isolated rod. The intensity of the far-field response of two widely spaced rods should be four times the response of an isolated rod. As shown in Fig. 7, the intensity of the far-field response for widely spaced rods oscillates (because of the retardation of the coupling) about the expected limit. For closely spaced rods, the strong coupling distorts the intrarod charge distribution. Charge induced at the gap by the incident field is strongly localized by the attraction between charges across the small gap. In reaction, the charge at the outside ends of the rods is drawn closer to the gap (see Figs. 6 and, later, 10), reducing the intrarod charge separation in the strongly coupled structure. As a result the dipole moment from the charges at the outside ends is $<Q(2 L+S)$ and the total dipole moment of the strongly coupled structure is less than the dipole moment of two widely spaced rods. This explains the decrease in far-field response as $S$ is reduced from the weakly coupled regime. The small increase in far-field response at the smallest $S$ indicates that the induced charge $\pm Q$ can increase when the coupling becomes strong enough.

The near-field enhancement in the gap is drastically increased when the gap is reduced to $2 \mathrm{~nm}$. The near-field enhancement is a factor of $\sim 300$ for the smallest gap. Heuristically, this is roughly the square of the near-field enhancement at the end of an isolated rod. One factor is due to the enhancement at the end of a rod, the second is due to the enhanced local field of the other rod that drives the first rod. For small $S$, the near-field enhancement is similar at all points on the rod axis in the gap. The enhancement decreases rapidly as $S$ increases because the coupling is weaker. The enhancement at midgap decreases even faster with increasing $S$ because the midpoint is further from the end of the rod.

For $S=20 \mathrm{~nm}$, the near-field enhancement for coupled rods is only a factor of 2 greater than for the isolated rod. Thus very small, nanometer-sized gaps are needed to get significant increases in the near field at the midpoint of the gap. In the context of a Raman experiment with the signal proportional to the fourth power of the field, ${ }^{2}$ the Raman signal from a molecule in middle of the smallest gap would be enhanced by $10^{10}$ relative to the Raman signal of a molecule in free space and by a factor of $10^{5}$ relative to a molecule at the end of an isolated rod.

Simply by looking at the far-field response, it would be difficult to distinguish whether a long-wavelength nearinfrared peak is due to a coupled pair of nanorods [see Fig. $5(\mathrm{a})]$ or whether the peak is due to a nanorod that is roughly twice as long [see Fig. 2(a)]. The near-field response distinguishes between these two possibilities. The near-field enhancement at the end of the nanorod is $\approx 30$ for a $500 \mathrm{~nm}$ long nanorod, whereas the pair of nanorods shows an enhancement of the near-field in the gap of $\approx 300$. Even though the energy (wavelength) of far- and near-field features can be similar, the near fields induced at the nanorods are much stronger in a coupled system. These differences are important for field-enhanced spectroscopies.

The feature just above $\Gamma_{\text {sphere, which is due to higher- }}$ order longitudinal and transverse modes, is also redshifted by the depolarization induced by the coupling and charge localization at the gap (see Fig. 5). However, the redshift is smaller than for the long-wavelength peak. The longwavelength peak is more sensitive to $S$. Charge piles up at 

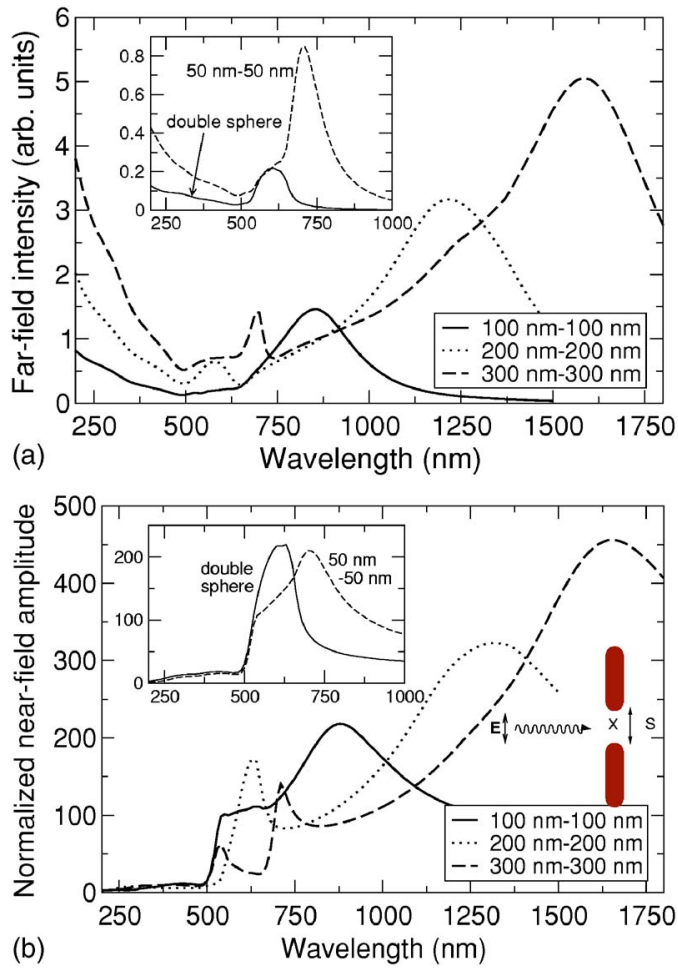

FIG. 8. (a) Far-field intensity as a function of wavelength for a plane wave incident on a pair of identical cylindrical Au nanorods with $S=2 \mathrm{~nm}$ and the indicated lengths $L . R=40 \mathrm{~nm}$. The polarization is parallel to the rod. (b) Normalized near-field amplitude at the midpoint between two nanorods for the same geometry as in (a). The insets show the results for small $L$ and for two coupled spheres.

the gap mainly in a dipolar pattern for the long-wavelength response (see the following discussion on modes), which can strongly reduce the intrarod restoring forces and redshift the response. However, the feature near $\Gamma_{\text {sphere }}$ is connected with more transverselike modes with oscillations of the charge at the round ends of the rods and with higher-order longitudinal modes. Both types of modes are less effective at supporting charge localization at the gap and reducing the intrarod restoring forces. Still, the feature just above $\Gamma_{\text {sphere }}$ becomes more apparent and sharper in the coupled nanorods for $L$ $=200 \mathrm{~nm}$, even though it is not clearly shifted away from $\Gamma_{\text {sphere }}$ for the isolated rod with the same length, because of the redshift due to the coupling. Again, this feature in the far-field response is weakly enhanced by the coupling. The enhancement of this feature is much greater for the near-field response of the coupled nanorods.

The response below $\Gamma_{\text {sphere }}$ due to the radiating plasmons and the bulklike response is essentially unchanged by the coupling. The near-field response below $\Gamma_{\text {sphere }}$ is very weak. The far-field response below $\Gamma_{\text {sphere }}$ is independent of the gap and roughly a factor of 4 greater than for an isolated rod, as would be expected if the radiated field at short wavelength is proportional to the total volume (or length) of the structure.

The dependence of the far- and near-field responses of two coupled, identical nanorods on rod length $L$ is shown in Fig. 8. The $L$-dependence of the feature positions $\Gamma_{n}$ is shown in Fig. 3. As for the isolated rods, the $\Gamma_{n}$ increase monotonically with increasing $L$ for the coupled rods. Fea-
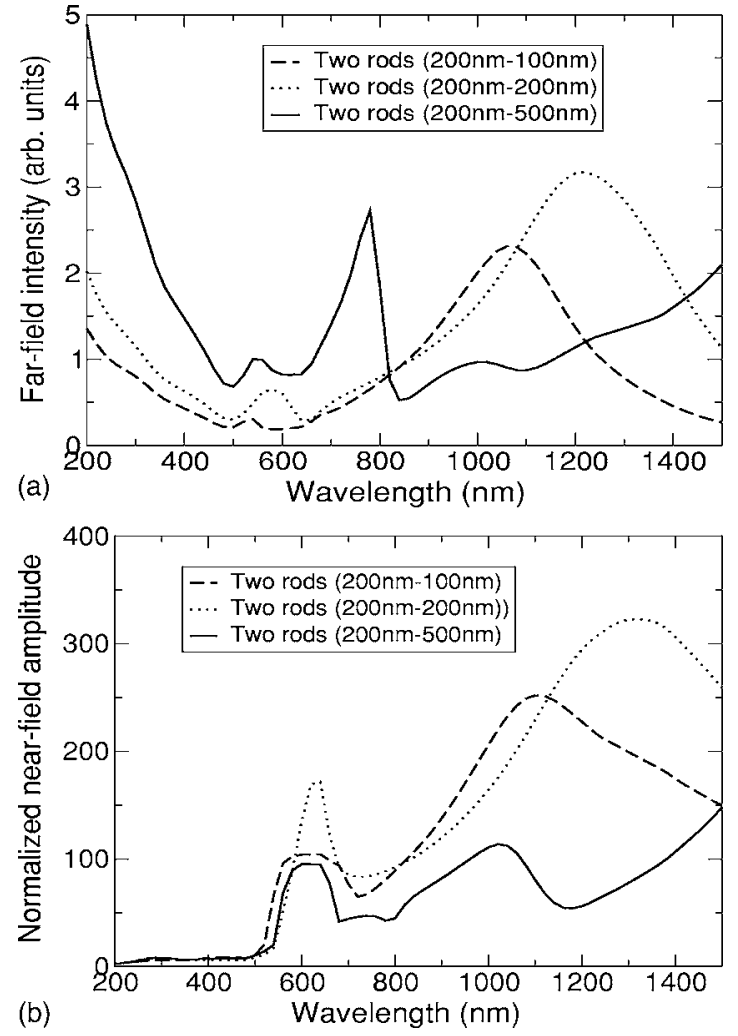

FIG. 9. (a) Far-field intensity as a function of wavelength for a plane wave incident on an asymmetric pair of cylindrical Au nanorods with $S=2$. The length of one nanorod is $200 \mathrm{~nm}$. $L$ for the second nanorod is varied as indicated. $R=40 \mathrm{~nm}$. The polarization is parallel to the rod. (b) Normalized near-field amplitude at the midpoint between two nanorods for the same geometry as in (a).

tures become sharper as $L$ increases because the modes are more widely separated. The long-wavelength far-field intensity increases monotonically with increasing $L$, whereas the far-field intensity below $\Gamma_{\text {sphere }}$ scales roughly as the volume of the coupled rods. For small $L$, the near-field enhancement at the long-wavelength peak actually decreases slightly for increasing $L$ (compare Figs. 2 and 8). For $L>150 \mathrm{~nm}$, the peak near-field enhancement increases monotonically with increasing $L$. This suggests that there are two regimes of interest. For small $L$, the field enhancement is not sensitive to the rod length, rather it is determined by rod termination (the lightning rod effect) ${ }^{49}$ For longer rods, the field enhancement is determined primarily by the strength of the dipole that can be excited along each nanorod and increases monotonically with increasing $L$.

Similar control of the far- and near-field responses can be achieved while keeping one nanorod fixed and varying the length of the second nanorod. The dependence of the response on rod length in asymmetric, coupled rods is shown in Fig. 9. One rod is fixed at a length of $200 \mathrm{~nm}$, while the length of the second rod is varied. The position of the nearinfrared peak can be tuned by varying the rod length, redshifting because of the reduction in intrarod restoring force that increases with rod length. The strength of the nearinfrared response also increases as the rod length increases. The middle peak is less sensitive to rod length. The far-field 
response at the shortest wavelengths scales with rod length, as expected for this bulklike response. For the far-field response, there does not appear to be any special length for optimal response. For the nearfield, the response at midwavelengths is optimal for rods of similar length.

The monotonic increase in $\Gamma_{n}$ for coupled, identical nanorods with increasing $L$ is shown in Fig. 3 . When the $\Gamma_{n}$ for the coupled nanorods are plotted versus the total length of the coupled nanorods, $L_{\text {tot }}=2 L$ rather than $L$ (not shown in Fig. 3), then the two regimes of behavior can again be seen. For small $L_{\text {tot }}$, the $\Gamma_{n}$ for the coupled nanorods are still redshifted from the $\Gamma_{n}$ for an isolated nanorod with $L=L_{\text {tot }}$. This indicates that the additional redshift due to the depolarization of the intrarod oscillations by the coupling between charges on the opposite sides of the gap is larger than the additional redshift in an isolated rod due to the reduction of the intrarod restoring force. For larger $L_{\text {tot }}$, the $\Gamma_{n}$ for the coupled nanorods is blueshifted from $\Gamma_{n}$ for an isolated nanorod with $L$ $=L_{\text {tot }}$. Reduction of the intrarod restoring force because of increasing rod length determines the redshift for isolated rods. For coupled nanorods with large $L_{\text {tot }}$, the reduction of the intrarod restoring force is again the dominant effect, but the interrod coupling makes this reduction less significant for a coupled nanorod pair with total length $L_{\text {tot }}$ than for an isolated nanorod with the same length. As discussed previously (see Fig. 6), the charges at the outside ends of the two coupled rods are closer together than they would be if there were no coupling, that is, in an isolated rod with $L=L_{\text {tot }}$. As a result, the reduction of the restoring forces due to increasing length is less in the coupled structure than in the isolated structure with the same total length.

The far-field response provides a direct measure of the dipole moment induced by the incident field. A comparison of the far-field response for isolated and coupled rods with the same total length (see Figs. 2, 8, and 9) again reveals the two regimes of behavior for fixed $S$. For large $L_{\text {tot }}$, the farfield response of an isolated rod is larger than the far-field response for the coupled structure with the same $L_{\text {tot }}$. As mentioned before, because of the coupling, the separation between the outside charges in a coupled structure is less, for large $L_{\text {tot }}$, than in a isolated rod with the same total length. This provides a smaller dipole moment. For small $L_{\text {tot }}$, the far-field response of an isolated rod is smaller than the farfield response for the coupled structure with the same $L_{\text {tot }}$. This indicates that more charge is polarized in short coupled structures than in the isolated rods with the same total length. As mentioned previously, increased charge polarization also explains the $S$ dependence of the far-field intensity in the limit of very strong coupling (Fig. 7).

Isolated and coupled rods exhibit a similar response. The primary differences are the splitting of degenerate modes, the coupling-induced intrarod charge distortion and reduction of intrarod restoring forces, and the resulting redshifts of features and drastic enhancements of the near field in the coupled structures. These differences occur because the modes are significantly modified by the coupling. As shown in Fig. 4, the modes for an isolated rod are either dipole active with surface-charge distributions that are antisymmetric about the rod center with an odd number of nodes in the charge distribution or symmetric (dipole-inactive) with an even number of nodes. This symmetry is broken when two rods are coupled together. The modes of a pair of coupled nanorods are shown in Fig. 10 for $m=0$. For two coupled identical rods, the surface-charge distribution of a mode is either symmetric or antisymmetric about the midpoint of the gap. Moreover, the modes of the coupled system should be formed from symmetric and antisymmetric combinations of the modes of the isolated rod. Figure 10 shows that this is the case.

Three different types of modes can be distinguished. First, a branch of antisymmetric, coupled modes is associated with strong, attractive coupling of the localized charge at the gap between the nanorods (modes $0 \mathrm{a}, 1 \mathrm{a}, 2 \mathrm{a}, \ldots$ in Fig. 10). These modes pile up a very high surface-charge density of opposite sign on the adjacent nanorods surfaces at the gap, forming a strong dipolarlike distribution at the gap, as happens for two coupled spheres. ${ }^{16}$ As for mode 0 of an isolated rod, mode $0 \mathrm{a}$ is unphysical (unless charge tunneling between the rods is possible) because this mode has a finite charge on each rod. All higher modes are charge neutral on each rod. Because of the strong attractive interaction between the opposite charges across the gap, there is significant reduction of intrarod restoring forces and redshift of these modes. These modes can be characterized by the number of nodes per rod. As shown on the left-hand side of Fig. 10, those modes with an odd number of nodes per rod are, effectively, symmetric combinations of the antisymmetric modes of the isolated rod. However, these modes are not strict symmetric combinations of the corresponding rod modes. There is a large distortion of the intrarod mode and charge pileup at the gap. Moreover, the nodes in the charge distribution shift toward the gap because of the charge pileup. These modifications of the charge distribution break the intrarod symmetry. Those modes with an even number of nodes per rod are antisymmetric combinations of the symmetric modes of the isolated rod. These modes also exhibit the charge pileup at the gap, and the shifting of the nodes are indicative of the broken intrarod symmetry.

There is a second group of modes with zero local dipole at the gap and in the entire structure because they are symmetric about the gap midpoint, as shown on the right-hand side of Fig. 10. These coupled modes are symmetric combinations of the symmetric modes of the isolated rod and antisymmetric combinations of the antisymmetric modes of the isolated rod. This group of symmetric coupled modes splits into two classes of modes, one class of modes at long wavelength above the surface plasmon wavelength $\lambda_{S}$ and another class at short wavelengths below $\lambda_{S}$. Because these modes are symmetric about the midgap, they have like charges at the two sides of the gap. For the modes at long wavelength (modes 0s [the unphysical mode with net intrarod charge], $1 \mathrm{~s}, 2 \mathrm{~s}, \ldots$ in Fig. 10), the buildup of charge at the gap is suppressed by the repulsive interaction between the charges across the gap. As a result, the coupling is weak. These modes are redshifted less by the coupling. As for the isolated rod, the mode wavelengths for both symmetric and antisymmetric modes above $\lambda_{S}$ decrease toward $\lambda_{S}$ as the number of nodes along the rod increases.

Because the symmetric modes above $\lambda_{S}$ have no charge buildup at the gap, there must be other symmetric modes 


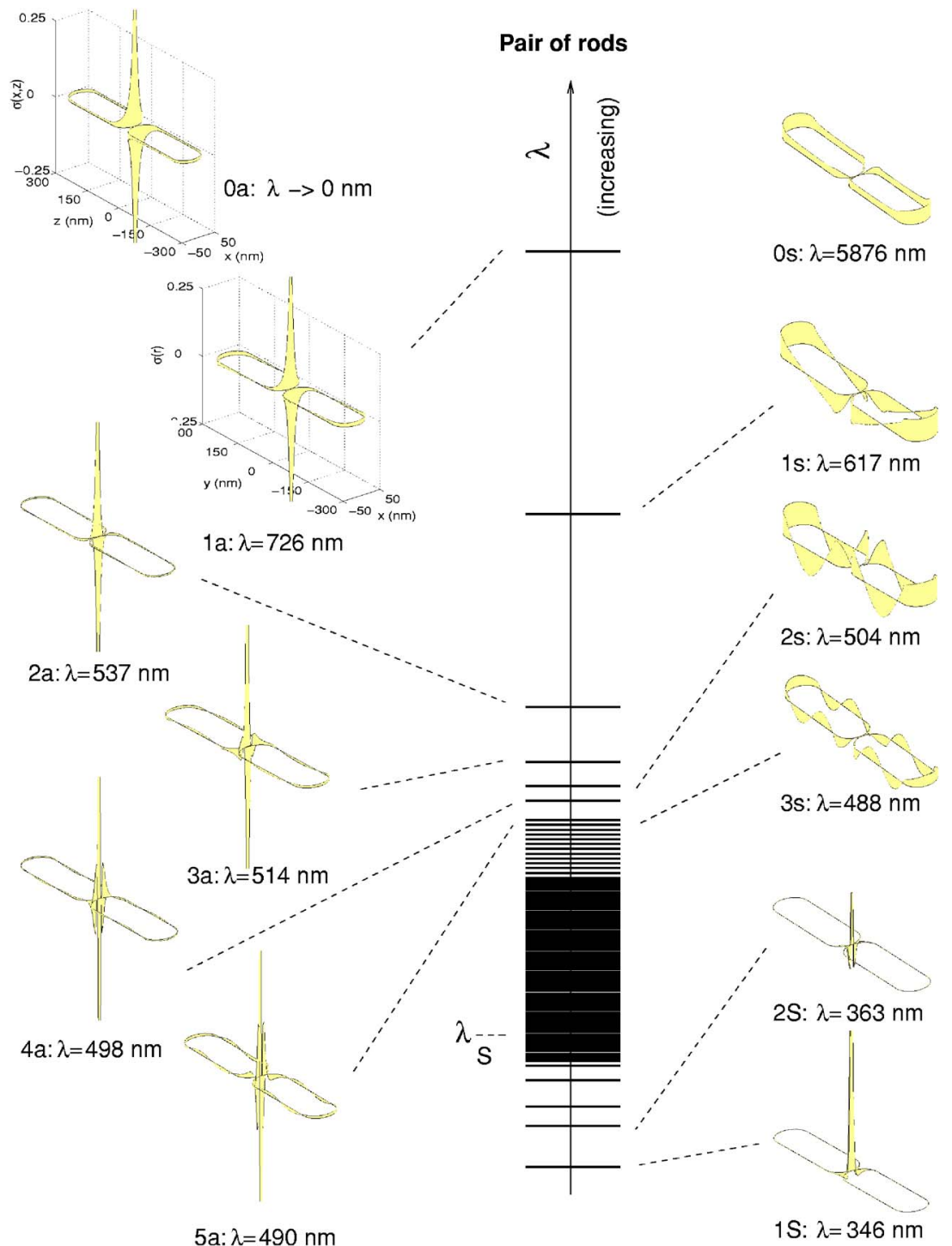

FIG. 10. Cross sections of the surface-modes surface-charge density in a pair of coupled gold nanorods, each with $L=200 \mathrm{~nm}$. $S=2 \mathrm{~nm}$, and $R=40 \mathrm{~nm}$. These modes have $m=0$ azimuthal number. with charge buildup around the gap. These modes (modes $1 \mathrm{~S}, 2 \mathrm{~S}, \ldots$ in Fig. 10) are blueshifted below $\lambda_{S}$ because of the large repulsive Coulomb energy of the charge at the gap. These modes approach $\lambda_{S}$, increasing in wavelength, as the number of nodes along the rod increases. Because of the large buildup of like charge on each side of the gap, there is also a pileup of compensating charge near the gap, as indicated by the rapid charge oscillation on the rod ends near the gap.

The modes most effectively excited by a plane wave polarized along the rod axis are those modes in the antisymmetric branch with a large net dipole moment. Modes with azimuthal number $m=1,2, \ldots$ can also be excited by electromagnetic waves. The structure of these modes is similar to $m=0$ modes with the additional $\cos (m \phi)$ dependence around the rod axis.

Another indication of the transverse or longitudinal character of the modes emerges from the different responses of these modes to different incident polarizations. Similar response to polarization perpendicular to the rod is observed when the plane wave is incident parallel or perpendicular to the rod. The near-infrared response to perpendicular polar- ization vanishes. Only the shorter wavelength feature remains in the response above $\Gamma_{\text {sphere }}$, blueshifted with respect to the response for parallel polarization. The blueshift of this shorter wavelength response to perpendicular polarization indicates that the transverse modes being excited are closer to $\Gamma_{\text {sphere }}$ than the higher-order longitudinal modes that are excited by parallel polarization. Most importantly, the nearfield enhancement is approximately 3 orders of magnitude smaller for perpendicular polarization.

In many experimental setups, such as the one presented in Fig. 1, the orientation of the metallic nanostructures will be random. This strong polarization dependence of the nearfield response should be considered when analyzing enhancement factors needed for field-enhanced spectroscopy, since the wrong polarization or spatial distribution of the rods could significantly reduce or shift the enhanced response.

\section{FACTORS INFLUENCING THE NEAR-FIELD SIGNAL}

Thus far, we have considered isolated rods and pairs of gold nanorods in vacuum. As shown in Fig. 1, the nanorods 


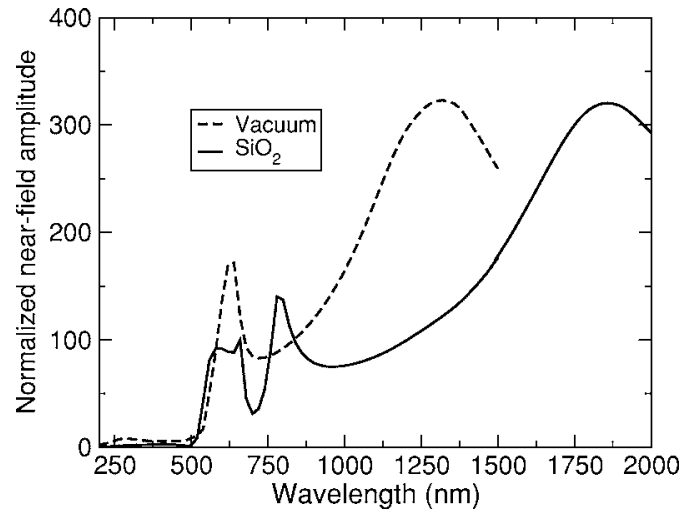

FIG. 11. Comparison of the normalized near-field amplitude as a function of wavelength for a plane wave incident on a pair of cylindrical gold nanorods surrounded by vacuum and by $\mathrm{SiO}_{2}$. The incident wave is polarized along the nanorod axis. $L=200 \mathrm{~nm}, R$ $=40 \mathrm{~nm}$, and $S=2 \mathrm{~nm}$.

may be surrounded by a dielectric environment. In this section we study how the choice of environment, nanorod material, and nanorod aspect ratio influence their response.

\section{A. Environment}

We first consider a pair of gold nanorods, $(L=200 \mathrm{~nm}$, $R=40 \mathrm{~nm}, S=2 \mathrm{~nm}$ ) embedded in $\mathrm{SiO}_{2}$, which is a common experimental situation. The dielectric data for $\mathrm{SiO}_{2}$ is taken from the literature. ${ }^{44}$ In Fig. 11 we show the near-field response with and without the $\mathrm{SiO}_{2}$ embedding medium. The dominant effect of the embedding medium is to further redshift the long wavelength peak. The shift is significant, with the peak shifting from 1300 to $1850 \mathrm{~nm}$. Moreover, the peaks at shorter wavelengths also redshift more, with additional peaks shifting above $\Gamma_{\text {sphere }}$ when the nanorods are embedded in $\mathrm{SiO}_{2}$. This trend is also present in the far-field spectrum (not shown) with peaks slightly redshifted when the pair of rods are embedded in $\mathrm{SiO}_{2}$. The screening by $\mathrm{SiO}_{2}$ does not noticeably reduce the near-field enhancement for the long-wavelength excitations, although the actual position of the features can shift dramatically.

\section{B. Rod composition}

We calculated the response of two coupled, identical nanorods made of silver and of aluminum to see how the nearfield enhancement depends on the bulk dielectric response of the nanorods. We show the near-field enhancement of coupled nanorods made from $\mathrm{Au}, \mathrm{Ag}$, and $\mathrm{Al}$ in Fig. 12. The response for the three materials at long wavelength is similar. Comparing silver and gold, the peak is located at almost the same wavelength with nearly the same magnitude. In this region of the spectrum, the dielectric functions for gold and silver are similar. The long-wavelength peak position should only be weakly dependent on material response as long as the peak position is determined approximately by $L$. Differences arise in the shorter wavelength region because the positions of the plasmons for gold and silver spheres are different. For aluminum, the long wavelength peak is slightly

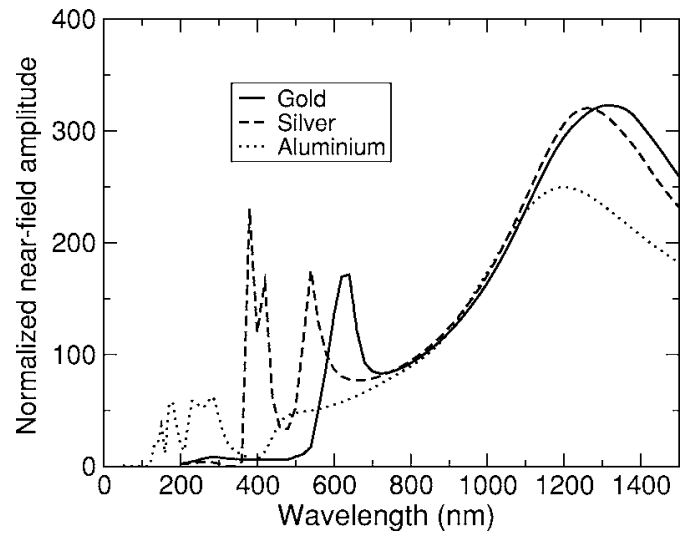

FIG. 12. Comparison of the normalized near-field amplitude as a function of wavelength for a plane wave incident on a pair of identical cylindrical nanorods for three different materials: gold, silver, and aluminum. The incident wave is polarized along the nanorod axis. $L=200 \mathrm{~nm}, R=40 \mathrm{~nm}$, and $S=2 \mathrm{~nm}$.

smaller in magnitude and is located at a slightly shorter wavelength. This shift is connected with the small wavelength for the plasmon in aluminum spheres, $(100-200 \mathrm{~nm})$. For these three materials, material response will not be the most significant feature for determining the enhancement, although the near-field response can be tuned some by material composition.

\section{Rod aspect ratio}

There is strong interest in finding nanostructures with the largest local enhancement. ${ }^{19}$ Coupling is an important source of field enhancement as our results show. The lightning-rod effect (using sharper ends to localize charge and concentrate local fields) also enhances the near-field response. In Fig. 13, we compare the response of coupled nanorods for radii $R$ $=10-60 \mathrm{~nm}$. The position of the long wavelength peak blueshifts from 1300 to $1200 \mathrm{~nm}$ as $R$ increases from 10 to $30 \mathrm{~nm}$ and then redshifts for further increases in $R$. This is summarized in Fig. 14. The same trend is seen in Fig. 13 for shorter wavelength features. Additional peaks appear above $\Gamma_{\text {sphere }}$ for rods with $R$ that produce large redshifts. The same trend is seen for both single rods and coupled rods. For $R>30 \mathrm{~nm}$, the charge becomes less localized at the end of the rod with increasing $R$, and the intrarod restoring force is reduced, producing the redshift. Because the restoring force is reduced with increasing $R$, the separation between charges increases and the dipole moment increases, producing an increase in the far-field response. For $R<30 \mathrm{~nm}$, the rods are too narrow and it becomes more difficult to sustain the charge at the end of the rod. This is seen in the more rapid decrease in the far-field intensity below for $R<30 \mathrm{~nm}$ and in the redshift with decreasing $R$ for $R<30 \mathrm{~nm}$. The near-field response increases monotonically with decreasing $R$, indicating that the lightning-rod effect due to tighter charge localization at smaller $R$ is the most important effect determining the $R$ dependence of the strength of the field near the end of a rod.

The long-wavelength enhancement of the near field increases from 300 to 500 because of the lightning-rod effect 

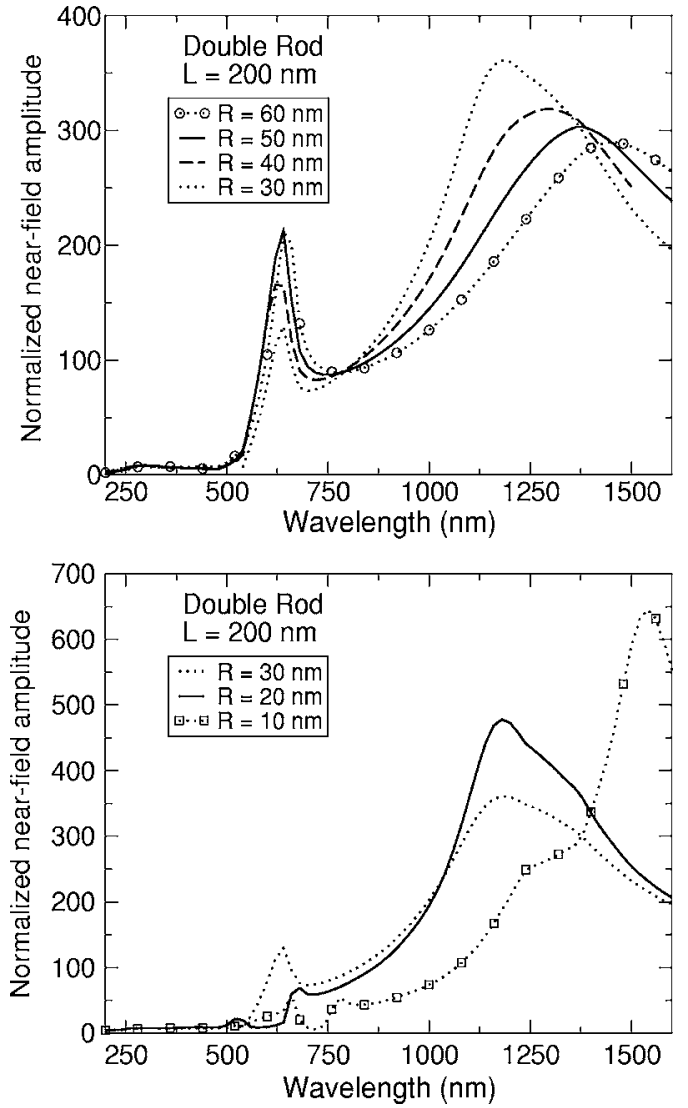

FIG. 13. Comparison of the normalized near-field amplitude as a function of wavelength for a plane wave incident on a pair of cylindrical gold nanorods for different radii. Each nanorod is $200 \mathrm{~nm}$ long. The polarization is along the nanorod axis and the near-field is determined at midgap for $S=2 \mathrm{~nm}$.

as $R$ decreases to $20 \mathrm{~nm}$. This near-field enhancement provides an order of magnitude increase in the signal for fieldenhanced spectroscopies. ${ }^{16}$ Damping because of surface scattering should be included for smaller structures. ${ }^{37}$ This damping could suppress additional local-field enhancement because of size reduction. ${ }^{50}$ However, recent work suggests that surface scattering is not significant in single small spheres $^{26}$ or spherical nanoshells ${ }^{51}$ and that damping is suppressed in nanorods. ${ }^{38}$ We assess the possible contribution of surface scattering to the response of small structures by performing calculations both with and without the additional damping. ${ }^{50}$ Additional near-field enhancement to 650 as $R$ decreases to $10 \mathrm{~nm}$ is predicted when the bulk response of $\mathrm{Au}$, without the additional surface scattering, is used to model the response of the rods (Fig. 14). When surface damping is included, the resonance wavelengths do not change much. However, reduction of $R$ from 20 to $10 \mathrm{~nm}$ has limited effect on the near-field enhancement in these coupled structures when the surface damping is included. ${ }^{52} \mathrm{~A}$ full understanding of the effect of surface scattering will be needed for determining the maximum field enhancement that is achievable using sharp structures.

\section{SUMMARY}

We have studied the optical response of coupled gold nanorods to investigate their potential for use in field-enhanced
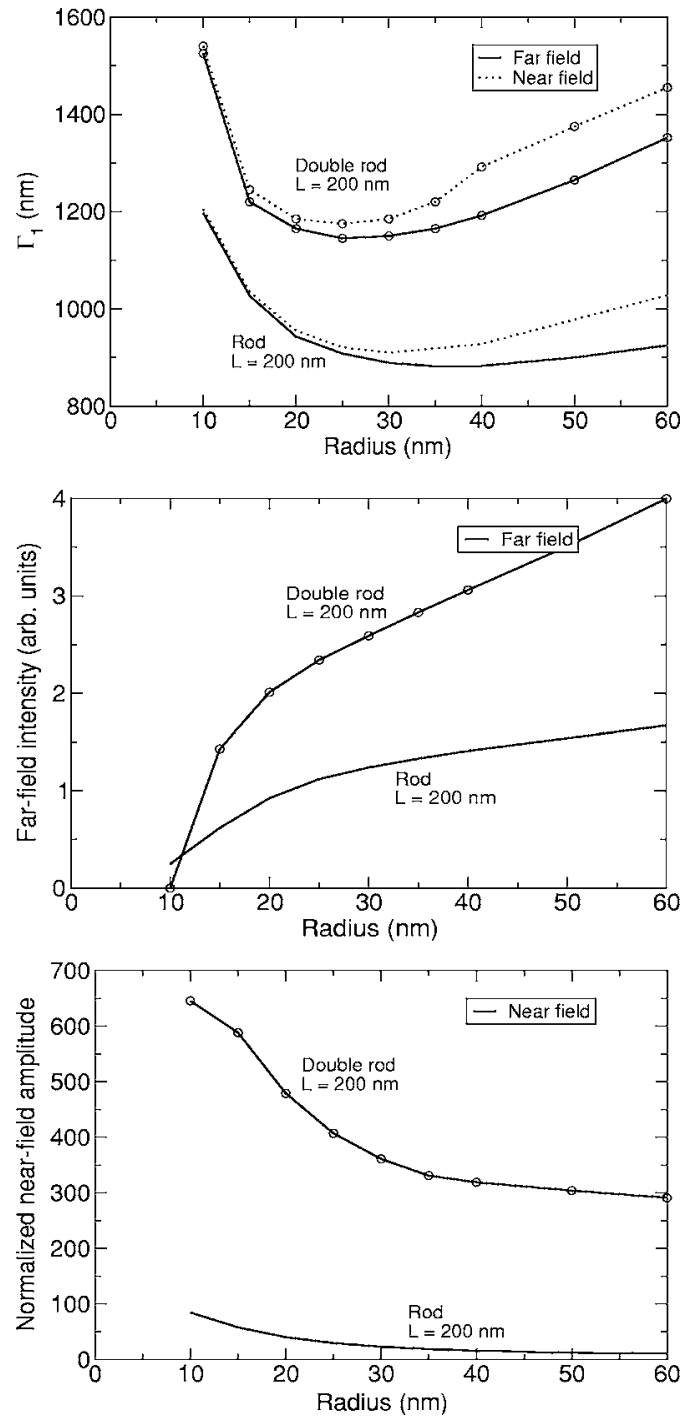

FIG. 14. Dependence of the far- and near-field response of a pair of identical coupled nanorods on the rod radius $R$ for a plane wave incident with polarization parallel to the rods. $L=200 \mathrm{~nm}, S$ $=2 \mathrm{~nm}$. The peak wavelength $\Gamma_{1}$ for the dipolar response, the farfield intensity and the normalized near-field intensity at midgap are shown. The corresponding results for an isolated rod are shown.

spectroscopy. We have analyzed the intrinsic modes of isolated rods and pairs of coupled rods, and calculated their response in the near and far field. For both isolated and coupled rods, we can distinguish three different wavelength regimes in the response: the long-wavelength infrared regime connected with the response of the lowest longitudinal mode; an intermediate regime, just above the spherical plasmon limt $\Gamma_{\text {sphere, }}$ which is connected with transverse and higherorder longitudinal modes; and a low-wavelength regime below $\Gamma_{\text {sphere }}$ connected with even higher-order modes, radiating plasmons, and interband transitions. The similar response of isolated and coupled nanorods masks significant differences in the local response that produces the near fields. For isolated rods, the response above $\Gamma_{\text {sphere }}$ redshifts with increasing rod length because the increased charge separation reduces the intrarod restoring force that drives the charge 
oscillation. The near and far-field responses at long wavelength increase with increasing rod length because the induced dipole moment is larger. Coupling pairs of nanorods further redshifts the response and dramatically enhances the near-field response in the gap. The gap in a coupled system breaks the intrarod symmetry of an isolated rod, and, as a consequence, the intrarod charge distribution is distorted and strong localization of charges can occur at the ends of the rods that form the gap. Strong attractive interaction between these charges across the gap reduces the intrarod restoring forces and provides this additional redshift because of coupling. The dramatic increases in the near-field enhancement for coupled structures result from this strong interaction of charges across the gap.

Coupled structures should be critical for use in fieldenhanced spectroscopies because near fields in a gap can be orders of magnitude higher than those at the end of isolated structures. In coupled nanorod structures, rod length, rod radius, and interrod gap are each important length scales. The response of coupled structures made from short rods is determined mostly by end effects, that is, how the charge localizes at the ends of the rods that define the gap. For longer rods, that response is determined primarily by rod length. The simplest way to optimize these structures to obtain enhanced fields is to increase rod length. However this comes at a significant cost because the position of the response redshifts monotonically with increasing rod length. Reducing rod radius can lead to significant increases in near-field en- hancement. However, there is a rod radius that provides the long-wavelength response at the smallest wavelength. For other radii, the response is redshifted. Moreover, the nearfield enhancement that can be achieved by using sharper rods may be limited if surface scattering effects that increase plasmonic damping become important in small structures. Reducing the gap also significantly enhances the local field, but again with a redshift of the response. Changing the environment that the nanorods are embedded in or changing the nanorod material can shift the response of the structures but does not dramatically change the near-field enhancement.

The response of the rods (both isolated and coupled pairs) is sensitive to the polarization of the incident wave. Polarization parallel to the rod axis provides the maximum enhancement, whereas polarization perpendicular to the rod generates weak near fields, exciting primarily transverse modes near $\Gamma_{\text {sphere }}$. As a consequence, the field polarization must be carefully controlled for any use of these structures in field-enhanced spectroscopy.

Nanorods can be considered as elongated versions of spheres that provide increased flexibility for tuning local response for use in field-enhanced spectroscopy, via the rod length, rod sharpness, and interrod coupling. Using fabrication methods, such as template synthesis, opens up the possibility of fabricating complex heterostructures made from multiple metals. Further study will be needed to see how this can be exploited for field-enhanced spectroscopy.
*Email: aizpurua@sc.ehu.es

†Email: garnett.bryant@nist.gov

${ }^{1}$ R. R. Chance, A. Prock, and R. Silbey, J. Chem. Phys. 60, 2744 (1974).

${ }^{2}$ Surface Enhanced Raman Scattering, edited by R. K. Chang and T. E. Furtak (Plenum, New York, 1982).

${ }^{3}$ J. I. Gersten and A. Nitzan, J. Chem. Phys. 73, 3023 (1980).

${ }^{4}$ A. Hartschuh, E. J. Sanchez, X. S. Xie, and L. Novotny, Phys. Rev. Lett. 90, 095503 (2003).

${ }^{5}$ H. Xu, E. J. Bjerneld, M. Käll, and L. Börjesson, Phys. Rev. Lett. 83, 4357 (1999).

${ }^{6}$ S. Nie and S. R. Emory, Science 275, 1102 (1997).

${ }^{7}$ K. Kneipp, Y. Wang, H. Kneipp, L. T. Perelman, I. Itzkan, R. R. Dasari, and M. S. Feld, Phys. Rev. Lett. 78, 1667 (1997).

${ }^{8}$ A. M. Michaels, J. Jiang, and L. Brus, J. Phys. Chem. B 104, 11965 (2000).

${ }^{9}$ J. Jiang, K. Bosnick, M. Maillard, and L. Brus, J. Phys. Chem. B 107, 9964 (2003).

${ }^{10}$ D. A. Genov, A. K. Sarychev, V. M. Shalaev, and A. Wei, Nano Lett. 4, 153 (2004).

${ }^{11}$ S. J. Oldenburg, J. B. Jackson, S. L. Wescott, and N. J. Halas, Appl. Phys. Lett. 75, 2897 (1999).

${ }^{12}$ R. C. Jin, Y. W. Cao, C. A. Mirkin, K. L. Kelly, G. C. Schatz, and J. G. Zheng, Science 294, 1901 (2001).

${ }^{13}$ N. Taub, O. Krichevski, and G. Markovich, J. Phys. Chem. B 107, 11579 (2003).

${ }^{14}$ J. J. Mock, S. J. Oldenburg, D. R. Smith, D. A. Schultz, and S.
Schultz, Nano Lett. 2, 465 (2002).

${ }^{15}$ T. R. Jensen, G. C. Schatz, and R. P. Van Duyne, J. Phys. Chem. B 103, 2394 (1999).

${ }^{16}$ H. Xu, J. Aizpurua, M. Käll, and P. Apell, Phys. Rev. E 62, 4318 (2000).

${ }^{17}$ B. Lamprecht, G. Schider, R. T. Lechner, H. Ditlbacher, J. R. Krenn, A. Leitner, and F. R. Aussenegg, Phys. Rev. Lett. 84, 4721 (2000).

${ }^{18}$ S. Coyle, M. C. Netti, J. J. Baumberg, M. A. Ghanem, P. R. Birkin, P. N. Bartlett, and D. M. Whittaker, Phys. Rev. Lett. 87, 176801 (2001).

${ }^{19}$ K. Li, M. I. Stockman, and D. J. Bergman, Phys. Rev. Lett. 91, 227402 (2003)

${ }^{20}$ K. H. Su, Q. H. Wei, X. Zhang, J. J. Mock, D. R. Smith, and S. Schultz, Nano Lett. 3, 1087 (2003).

${ }^{21}$ D. P. Fromm, A. Sundaramurthy, P. J. Schuck, G. Kino, and W. E. Moerner, Nano Lett. 4, 957 (2004).

${ }^{22}$ J. K. N. Mbindyo, T. E. Mallouk, J. B. Mattzela, I. Kratochvilova, B. Raxavi, T. N. Jackson, and T. S. Mayer, J. Am. Chem. Soc. 124, 4020 (2002).

${ }^{23}$ L. T. Cai, H. Skulason, J. G. Kushmeric, S. K. Pollack, J. Naciri, R. Shashidhar, D. L. Allara, T. E. Mallouk, and T. S. Mayer, J. Phys. Chem. B 108, 2827 (2004).

${ }^{24}$ G. C. Papavassiliou, Prog. Solid State Chem. 12, 185 (1980).

${ }^{25}$ G. Mie, Ann. Phys. 25, 377 (1908).

${ }^{26}$ T. Klar, M. Perner, S. Grosse, G. von Plessen, W. Spirkl, and J. Feldmann, Phys. Rev. Lett. 80, 4249 (1998). 
${ }^{27}$ K. L. Kelly, E. Coronado, L. L. Zhao, and G. C. Schatz, J. Phys. Chem. B 107, 668 (2003).

${ }^{28}$ J. P. Kottmann, O. J. F. Martin, D. R. Smith, and S. Schultz, Opt. Express 6, 213 (2000).

${ }^{29}$ K. Imura, T. Nagahara, and H. Okamoto, J. Phys. Chem. B 108, 16344 (2004).

${ }^{30}$ E. Prodan, C. Radloff, N. J. Halas, and P. Nordlander, Science 302, 419 (2003).

${ }^{31}$ J. Aizpurua, P. Hanarp, D. S. Sutherland, M. Käll, G. W. Bryant, and F. J. García de Abajo, Phys. Rev. Lett. 90, 057401 (2003).

${ }^{32}$ J. P. Kottmann and O. J. F. Martin, Opt. Lett. 26, 1096 (2001).

${ }^{33}$ W. Rechberger, A. Hohenau, A. Leitner, J. R. Krenn, B. Lamprecht, and F. R. Aussenegg, Opt. Commun. 220, 137 (2003).

${ }^{34}$ L. Zhao, K. L. Kelly, and G. C. Schatz, J. Phys. Chem. B 107, 7343 (2003).

${ }^{35}$ C. Rockstuhl, M. G. Salt, and H. P. Herzig, J. Opt. Soc. Am. A 20, 1969 (2003).

${ }^{36}$ E. Prodan and P. Nordlander, Nano Lett. 3, 543 (2003).

${ }^{37}$ S. Link and M. A. El-Sayed, J. Phys. Chem. B 103, 8410 (1999).

${ }^{38}$ C. Sönnichsen, T. Franzl, T. Wilk, G. von Plessen, J. Feldmann, O. Wilson, and P. Mulvaney, Phys. Rev. Lett. 88, 077402 (2002).

${ }^{39}$ F. Pincemin, A. Sentenac, and J. J. Greffet, J. Opt. Soc. Am. A 11, 1117 (1994).
${ }^{40}$ R. Ruppin, Electromagnetic Surface Modes, edited by A. D. Boardman (Wiley, New York, 1982), pp. 349.

${ }^{41}$ Bo E. Sernelius, Surface Modes in Physics (Wiley, Berlin, 2001).

${ }^{42}$ F. J. García de Abajo and A. Howie, Phys. Rev. Lett. 80, 5180 (1998); Phys. Rev. B 65, 115418 (2002).

${ }^{43}$ F. J. García de Abajo and J. Aizpurua, Phys. Rev. B 56, 15873 (1997).

${ }^{44}$ E. D. Palik, Handbook of Optical Constants of Solids (Academic, New York, 1985).

${ }^{45}$ N. Yamamoto, K. Araya, and F. J. García de Abajo, Phys. Rev. B 64, 205419 (2001).

${ }^{46} \mathrm{H}$. Raether, Excitation of Plasmons and Interband Transitions by Electrons (Springer-Verlag, Berlin, 1980).

${ }^{47}$ P. B. Johnson and R. W. Christy, Phys. Rev. B 6, 4370 (1972).

${ }^{48}$ M. I. Stockman, Phys. Rev. Lett. 93, 137404 (2004).

${ }^{49}$ G. W. Bryant and J. Aizpurua (unpublished). Calculations for coupled rods with different terminations (flat ends or concave ends) show very different response for small $L$. For larger $L$, the response of rods with different terminations becomes more similar.

${ }^{50}$ E. J. Zeman and G. C. Schatz, J. Phys. Chem. 91, 634 (1987).

${ }^{51}$ C. L. Nehl, N. K. Grady, G. P. Goodrich, F. Tam, N. J. Halas, and J. H. Hafner, Nano Lett. 4, 2355 (2005).

${ }^{52}$ A. Schwarzkopf, G. W. Bryant, and J. Aizpurua (unpublished). 